\title{
Schisandrin ameliorates cognitive impairment and attenuates $A \beta$ deposition in APP/PS1 transgenic mice: involvement of adjusting neurotransmitters and their metabolite changes in the brain
}

\author{
Bin-bin WEI, Ming-yan LIU, Zai-xing CHEN, Min-jie WEI* \\ School of Pharmacy, China Medical University, Shenyang 110122, China
}

\begin{abstract}
Neurotransmitters (NTs) in the brain are involved in neurodegenerative diseases, such as Alzheimer's disease (AD). Schisandrin is a major ingredient of Schisandra chinensis (Turcz.) Baill and has been used for the treatment of AD. In this study we examined the therapeutic effects of schisandrin in APP/PS1 transgenic mice, and correlated the beneficial effects on cognitive impairment with the adjustments in NTs and their metabolites in the mouse brains. APP/PS1 mice were treated with schisandrin $\left(2 \mathrm{mg} \cdot \mathrm{kg}^{-1} \cdot \mathrm{d}^{-1}, \mathrm{ip}\right)$ for 2 weeks. In Morris Water Maze test; untreated APP/PS1 mice displayed significant cognitive impairment compared with normal mice; schisandrin administration ameliorated the cognitive impairment and significantly decreased A $\beta$ deposition in the hippocampus. In order to assess the effects of schisandrin on NTs and their metabolites, we developed a rapid and sensitive UPLC-MS/MS method for simultaneous determination of serotonin, 5-hydroxyindole acetic acid, dopamine, norepinephrine, y-aminobutyric acid, glutamic acid, homovanillic acid, 3,4-dihydroxyphenylacetic acid and acetylcholine in mouse brains. This method conformed to methodology validation requirements. We found that there were statistically significant differences in these NTs and their metabolites between untreated APP/ PS1 mice and normal mice, whereas schisandrin administration restored the abnormal NTs and their metabolites levels. These results suggest that schisandrin could alter the levels of these NTs and their metabolites in the brain, thus ameliorating learning and memory impairments in APP/PS1 mice.
\end{abstract}

Keywords: Alzheimer's disease; APP/PS1 transgenic mice; schisandrin; learning and memory; A $\beta$ deposition; neurotransmitters; UPLC-MS/MS

Acta Pharmacologica Sinica (2018) 39: 616-625; doi: 10.1038/aps.2017.135; published online 11 Jan 2018

\section{Introduction}

Alzheimer's disease (AD) is a progressive neurodegenerative disorder that is characterized by cognitive deficits and behavior disorders, and AD mainly occurs in people over sixtyfive years old ${ }^{[1-4]}$. AD worsens as it progresses and eventually leads to death. Currently, AD affects approximately 44 million people and will lead to increasingly significant economic burdens on society ${ }^{[5]}$. The major pathogenesis of AD includes the cholinergic hypothesis and the amyloid cascade hypothesis ${ }^{[6,7]}$. The core feature of the amyloid cascade hypothesis is the formation of the $\beta$-amyloid peptide $(A \beta)$, which causes neurotoxic effects through oxidative stress, calcium overburden, and neuronal apoptosis as well as memory impairment.

\footnotetext{
* $T o$ whom correspondence should be addressed.

E-mail minjie_wei@163.com

Received 2017-07-02 Accepted 2017-09-18
}

There are various reports about the etiology of AD, and some of them involve neurotransmitters $(\mathrm{NTs})^{[8-10]}$. NTs and their metabolites in the central nervous system (CNS) are known to play a significant role in transmitting signals, and the disordered alteration of NTs is a pathological characteristic of AD. Therefore, the quantification of NTs and their metabolites, which are distributed widely throughout the CNS, would have important clinical value in the development of AD treatment ${ }^{[11-13]}$. NTs can be classified as monoamines, amino acids and others, such as monoamine NTs that consist of serotonin (5-HT), 5-hydroxyindole acetic acid (5-HIAA), acetylcholine (ACh), dopamine (DA), norepinephrine (NE), 3, 4-dihydroxyphenylacetic acid (DOPAC), and homovanillic acid (HVA) as well as amino acid NTs, including $\gamma$-aminobutyric acid (GABA) and glutamic acid (Glu). Their molecular structures are shown in Figure 1. Despite its complex pathogenesis, AD was widely accepted as being associated with changes in the 

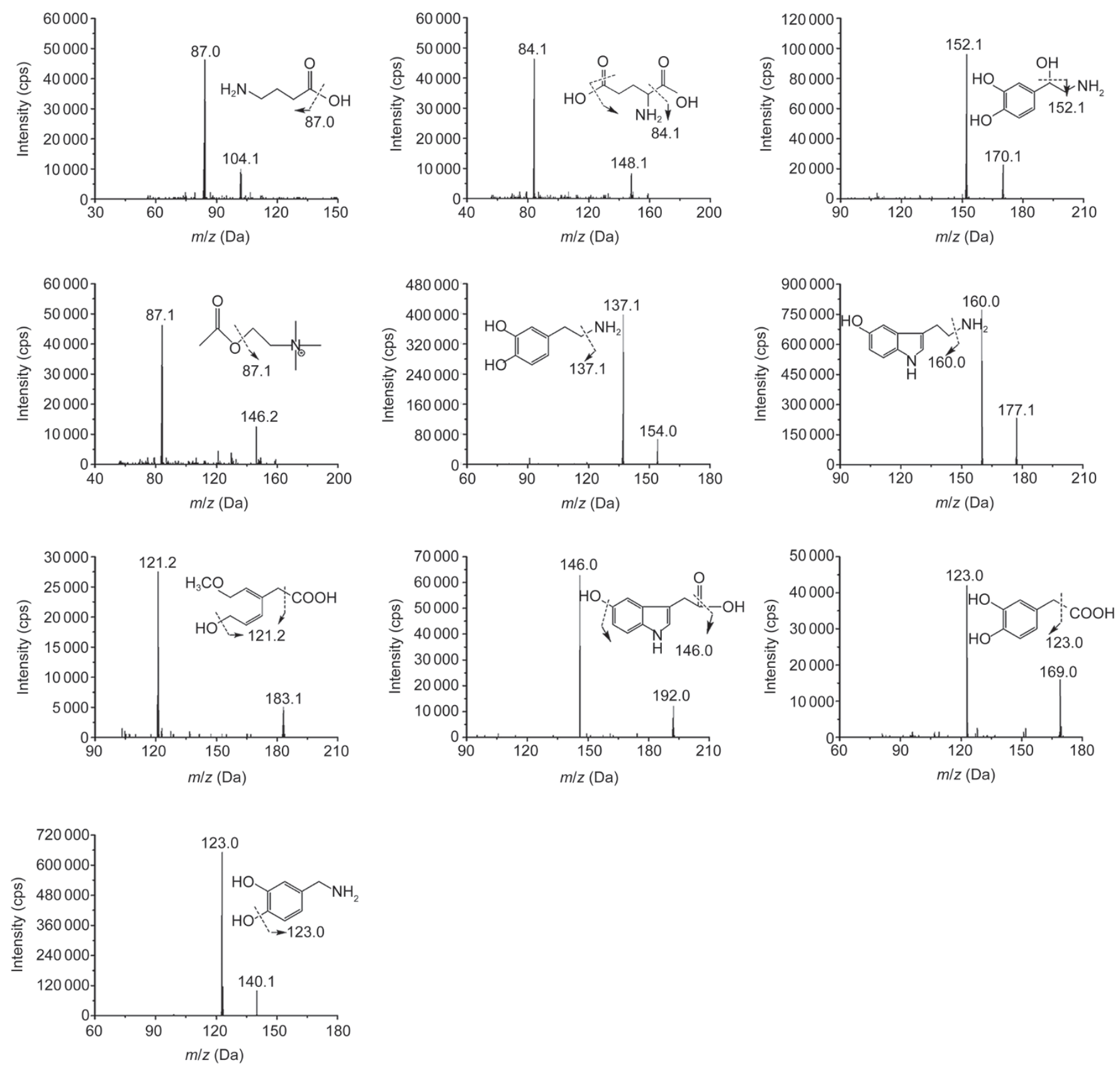

Figure 1. Chemical structures and MS/MS scan product ion spectrums of the NTs and their metabolites investigated together and internal standard. GABA (A), Glu (B), NE (C), ACh (D), DA (E), 5-HT (F), HVA (G) 5-HIAA (H), DOPAC (I) and DHBA (J; IS).

levels of NTs and their metabolites in the brain. The concentrations of these NTs in the brain are important biomarkers for disease states and drug effects ${ }^{[1,15]}$.

Therefore, in the pharmacotherapy of $\mathrm{AD}$, attempts to restore the dysfunction of transmitter systems should be a feasible treatment strategy. Currently, only drugs that can improve the function of cholinergic neuron acetylcholinesterase (AChE) inhibitors have been successful, and the FDA has approved five AChE inhibitors, including donepezil, rivastigmine, galantamine, huperzine and tacrine, that have been widely used in clinical treatment for AD patients ${ }^{[16-19]}$.
However, due to adverse reactions and poor patient compliance, $\mathrm{AD}$ drug treatment can still be ineffective. Therefore, the demand for new and high-efficiency AD drugs is increasing. In traditional Chinese Medicine (TCM), Schisandra chinensis (Turcz.) Baill is a common ingredient in prescriptions and could also be used alone to treat $\mathrm{AD}^{[20,21]}$. Schisandrin is one of the major active ingredients in $S$ chinensis that has been known to exert beneficial neuroprotective effects ${ }^{[22,23]}$. However, no study has been performed to evaluate whether schisandrin treatment ameliorates cognitive impairment in $\mathrm{AD}$ animals or to determine whether an ameliorating effect occurs through 
regulating the NT levels to restore the dysfunction of transmitter systems for $\mathrm{AD}$ treatment.

Therefore, in the present study, we used APP/PS1 transgenic mice as AD model animals. We first found that schisandrin ameliorates learning and memory effects by using the Morris water maze (MWM) test. Furthermore, we determined that schisandrin attenuates $A \beta$ deposition in the hippocampus and cortex of mice using immunohistochemistry. Finally, we developed an efficient, sensitive and selective UPLC-MS/MS method for the simultaneous determination of 5-HT, 5-HIAA, DA, DOPAC, HVA, NE, GABA, Glu and ACh in brain samples from mice to investigate the amelioration of learning and memory effects by schisandrin in AD mice. This study was the first to compare possible differences in the effects of these compounds between normal and $\mathrm{AD}$ mice. This result might be a useful tool for objective diagnosis of $A D$, which could benefit further neurological disease research.

\section{Materials and methods Reagents}

Schisandrin (purity $>99 \%$ ) was purchased from the National Institute for the Control of Pharmaceutical and Biological Products (Beijing, China). DA, DOPAC, HVA, NE, 5-HT, 5-HIAA, $\mathrm{ACh}$, Glu and GABA were purchased from Sigma (St Louis, MO, USA). The internal standard (IS) 3, 4-dihydroxybenzylamine (DHBA) was purchased from Alfa Aesar (Company Inc, USA).

HPLC grade acetonitrile and formic acid were purchased from Fisher Scientific (Fair Lawn, NJ, USA). Ether (HPLC grade) was provided by Shandong Yuwang Industrial Co Ltd (Yucheng, China). Distilled water prepared with demineralized water was used throughout the study.

\section{Instruments}

A WMT-100 MWM analysis system and a BI2000 image analysis system were purchased from Chengdu Taimeng Technology Co Ltd (Chengdu, China). An Olympus BX-61 microscope (Tokyo, Japan) was used, as was a 3500 MS/MS system from Applied AB Sciex (Foster City, CA, USA) coupled to an Agilent UPLC 1290 system (Agilent, CA, USA).

\section{Animals and treatment}

APP/PS1 double-transgenic mice (20-28 g weight) were obtained from The Jackson Laboratory ${ }^{[24,25]}$. C57 BL/6J mice (21-30 g weight) were used as normal mice. Males and females were equally distributed in each group. These mice were housed in cages in a controlled environment $\left(22-25^{\circ} \mathrm{C}\right.$, $55 \%$ relative humidity, $12 \mathrm{~h}$ light/dark cycle) with free access to food and water, and they were maintained in a specific pathogen-free environment at the Laboratory of Animal Science at China Medical University. All animal care and experimental procedures complied with the Standard Medical Laboratory Animal Care and Use Protocols (Ministry of Health PR China, 1998) and the Laboratory Animal Ethical Standards of China Medical University.

Twenty 12-month-old APP/PS1 transgenic mice were randomly and equally assigned to two groups, including the schisandrin-treated APP/PS1 group and the vehicle-treated APP/PS1 group (APP/PS1 group), with 10 mice in each group. Another $10 \mathrm{C} 57 \mathrm{BL} / 6 \mathrm{~J}$ mice were assigned as the normal group. Schisandrin was dissolved into distilled water at a concentration of $0.2 \mathrm{mg} / \mathrm{mL}$. Distilled water was used as the vehicle. The schisandrin-treated APP/PS1 group received schisandrin $\left(2 \mathrm{mg} \cdot \mathrm{kg}^{-1} \cdot \mathrm{d}^{-1}\right)$ intragastrically for 2 weeks, and the APP/PS1 and normal groups received the same volume of vehicle for 2 weeks.

\section{Morris water maze test}

The 3 groups of mice were observed in Morris water maze $(\mathrm{MWM})^{[26]}$ behavioral tests for 7 consecutive days, including navigation tests and a probe trial test, to study whether schisandrin treatment ameliorates learning and memory impairment in AD mice. The Morris water maze is a stainlesssteel circular water tank $(120 \mathrm{~cm}$ diameter $\times 50 \mathrm{~cm}$ in height) equipped with a platform $(10 \mathrm{~cm}$ diameter $)$ placed in the second quadrant and submerged $0.5-1 \mathrm{~cm}$ below the surface of the water. In brief, mice were allowed to swim freely for $1 \mathrm{~min}$ without the platform to adjust themselves to baseline circumstances on the baseline day (day 0). From the first day to the fifth day, the platform was placed under the water in the tank for navigation tests, and each mouse was subjected to four trials per day at intervals of $60 \mathrm{~s}$ for spatial acquisition. Different start locations were used for each trial. If a mouse failed to find the platform within $60 \mathrm{~s}$, it would be picked up and placed on the platform for $60 \mathrm{~s}$. For each trial, the latency and the length of the path through which the mice found the hidden platform were recorded. On the sixth day, a probe trial was performed to assess memory consolidation. In this trial, the platform was removed from the tank, and the mice were allowed to swim freely for $60 \mathrm{~s}$. The start position was a novel one that was $180^{\circ}$ from the original platform position to ensure that the spatial preference reflected the memory of the goal location rather than a preference for a specific swim path. The frequency with which each mouse crossed the center of the quadrant (where the platform was previously located) and the percentage of time that each mouse spent in the quadrant were recorded.

\section{Animal dissection and tissue collection}

Twenty-four hours after the behavioral tests, half of the mice $(n=5)$ in each group were intraperitoneally anesthetized with sodium pentobarbital $(50 \mathrm{mg} / \mathrm{kg})$ and then transcardially perfused with normal saline followed by $4 \%$ paraformaldehyde solution. Half of their brains were removed and post-fixed in $4 \%$ paraformaldehyde overnight at $4{ }^{\circ} \mathrm{C}$, and routine paraffin sections $(4 \mu \mathrm{m})$ were prepared for immunohistochemistry analysis. The remaining half of the brains in each group were rapidly removed, and the hippocampus and cerebral cortex were dissected out and stored at $-80^{\circ} \mathrm{C}$ for NT determination.

\section{Immunohistochemistry}

Next, immunohistochemistry was used to study whether schisandrin treatment reduced $A \beta$ deposits in the brains of $\mathrm{AD}$ mice. Sections were dewaxed in xylene and rehydrated 
in a series of graded alcohols. After dewaxing and rehydration, the sections were boiled in citrate buffer $(10 \mathrm{mmol} / \mathrm{L}, \mathrm{pH}$ 6.0) for $20 \mathrm{~min}$ using a microwave oven for antigen retrieval. Then, the sections were treated with $3 \% \mathrm{H}_{2} \mathrm{O}_{2}$ in $0.1 \mathrm{~mol} / \mathrm{L}$ phosphate buffered solution (PBS) for $20 \mathrm{~min}$ at room temperature to abolish endogenous peroxidase activity. After washing sections with PBS, the sections were blocked with normal goat serum at $37^{\circ} \mathrm{C}$ for $30 \mathrm{~min}$. Subsequently, the sections were incubated overnight at $4{ }^{\circ} \mathrm{C}$ with a primary rabbit anticaspase 3 antibody (1:500, Abcam) and an anti-A $\beta$ antibody (1:500, Thermo). After rinsing, the sections were incubated in biotinylated goat anti-rabbit IgG (1:200, Maixin) at $37^{\circ} \mathrm{C}$ for $30 \mathrm{~min}$, followed by streptavidin-peroxidase conjugate $(1: 200$, Maixin) at $37^{\circ} \mathrm{C}$ for $30 \mathrm{~min}$. The immunoreactions were visualized by staining sections with $3^{\prime}$-diaminobenzidine (DAB) for 1-3 min. Finally, the sections were counterstained with hematoxylin for $5 \mathrm{~min}$. Images were obtained using a microscope and digitized using an attached Spot flex imaging system (Tokyo, Japan).

\section{Determination of NTs in brain samples using UPLC-MS/MS Instruments and UPLC-MS/MS conditions}

The controls and samples were analyzed on a 3500 MS/MS system from Applied AB Sciex coupled to an Agilent UPLC 1290 system. Separations were accomplished on an Agilent Poroshell 120, SB-Aq $(2.1 \mathrm{~mm} \times 100 \mathrm{~mm}, 2.7 \mu \mathrm{m})$ with an Agilent guard cartridge at a temperature of $30{ }^{\circ} \mathrm{C}$. The mobile phase, which consisted of acetonitrile (solvent $\mathrm{A}$ ) and $0.1 \%$ formic acid in water (solvent B) was delivered at a flow rate of 0.3 $\mathrm{mL} / \mathrm{min}$. The linear gradient elution program was performed as follows: (1) held at 95\% B for 0.1-2.0 min; (2) from 95\% B to $20 \%$ B for 2.0-5.0 min; (3) from 20\% B to 95\% B for 5.0-6.0 min; and (4) held at $95 \%$ B for $2.0 \mathrm{~min}$. The injection volume was $5 \mathrm{~mL}$, and the total time for the chromatographic run was 8.0 min per sample.

The mass spectrometer was operated in positive ion mode with a TurboIonSpray source. Table 1 represents the optimized MRM parameters for the analytes and the IS. The MS figures are shown in Figure 2. The Q1, Q3, declustering potential (DP) and collision energy (CE) values were based on

Table 1. Optimized multiple-reaction-monitoring (MRM) parameters for GABA, Glu, NE, ACh, DA, 5-HT, HVA, 5-HIAA, DOPAC and DHBA (IS).

\begin{tabular}{llllll}
\hline Analytes & Q1 (amu) & Q3 (amu) & DP $(\mathrm{V})$ & CE $(\mathrm{eV})$ & $t_{\mathrm{R}}(\mathrm{min})$ \\
\hline GABA & 104.1 & 87.0 & 30 & 15 & 0.83 \\
Glu & 148.1 & 84.1 & 40 & 18 & 0.90 \\
NE & 170.1 & 152.1 & 30 & 10 & 0.96 \\
ACh & 146.2 & 87.1 & 55 & 20 & 1.01 \\
DA & 154.0 & 137.1 & 20 & 15 & 1.12 \\
5-HT & 177.1 & 160.0 & 45 & 10 & 1.91 \\
HVA & 183.1 & 121.2 & 37 & 12 & 3.11 \\
5-HIAA & 192.0 & 146.0 & 55 & 20 & 5.12 \\
DOPAC & 169.0 & 123.0 & 25 & 12 & 4.21 \\
DHBA (IS) & 140.1 & 123.0 & 40 & 13 & 1.51 \\
\hline
\end{tabular}

Analyst software 1.5 from Applied Biosystems/MDS Sciex. The other ionization parameters were as follows: curtain gas (CUR), 20 (arbitrary units); ion source gas 1 (GS1), 50 (arbitrary units); ion source gas 2 (GS2), 50 (arbitrary units); source temperature (TEM), $500{ }^{\circ} \mathrm{C}$; and entrance potential (EP), $10 \mathrm{~V}$. The dwell time of each MRM transition was $50 \mathrm{~ms}$.

\section{Standard solution and quality control samples}

Individual stock solutions of NTs were prepared in acetonitrile/water mixed solution $(v / v, 1: 1)$. The brain tissue samples were homogenized in a four-fold excess volume of methanol. The brain tissue standards for each of the 9 analytes were prepared at concentrations of 2.0, 10, 50, 150, 250, 500 and 1000 $\mathrm{ng} / \mathrm{mL}$ for GABA, at concentrations of 4.0, 20, 100, 300, 500, 1000 and $2000 \mathrm{ng} / \mathrm{mL}$ for Glu and NE and at concentrations of 1.0, 5.0, 25, 75, 125, 250 and $500 \mathrm{ng} / \mathrm{mL}$ for DA, ACh, 5-HT, 5-HIAA, DOPAC and HVA. These standards were prepared by adding the appropriate amount of the standard working solutions to the blank brain tissue homogenates. Quality control samples were prepared in the same fashion. A working solution for the IS (200 ng/mL) was also prepared.

\section{Sample preparation}

One-hundred microliter brain samples were transferred to a $10-\mathrm{mL}$ centrifuge tube along with $10 \mu \mathrm{L}$ IS solution and $10 \mu \mathrm{L}$ water/acetonitrile $(45: 55, v / v)$. After the samples were vortexed for $30 \mathrm{~s}, 1 \mathrm{~mL}$ ether was added. The analytes and IS were extracted from brain or brain tissue homogenates by vortexing the homogenates for $5 \mathrm{~min}$ and shaking them for $5 \mathrm{~min}$. Then, the samples were centrifuged at $3000 \times g$ for $5 \mathrm{~min}$. The organic layer was quantitatively transferred to a 5 -mL glass tube and evaporated to dryness at $35^{\circ} \mathrm{C}$ under a slight stream of nitrogen. Then, the dried extract was reconstituted in 100 $\mu \mathrm{L}$ solvent (water-acetonitrile, $45: 55, v / v$ ), followed by an injection of a $2-\mu \mathrm{L}$ aliquot into UPLC-MS/MS for analysis.

\section{Method validation}

The method was fully validated according to US FDA guidelines and $\mathrm{ICH}^{[27,28]}$ with respect to selectivity, a lower limit of quantification (LLOQ), calibration curve, accuracy and precision, recovery, matrix effect and stability.

\section{Statistical analysis}

Data were expressed as the mean \pm SD or mean \pm SEM. All statistical analyses were performed using the SPSS statistical software package (Statistical Package for the Social Sciences, version 13.0, SPSS Inc, Chicago, IL, USA). Differences among normal, APP/PS1 and schisandrin-treated APP/PS1 groups were assessed by a one-way analysis of variance (ANOVA) followed by Tukey's post-hoc test. Differences were considered significant at $P<0.05$ and $P<0.01$.

\section{Results}

Schisandrin treatment ameliorated learning and memory impairment in APP/PS1 mice

To investigate whether schisandrin treatment improved learn- 

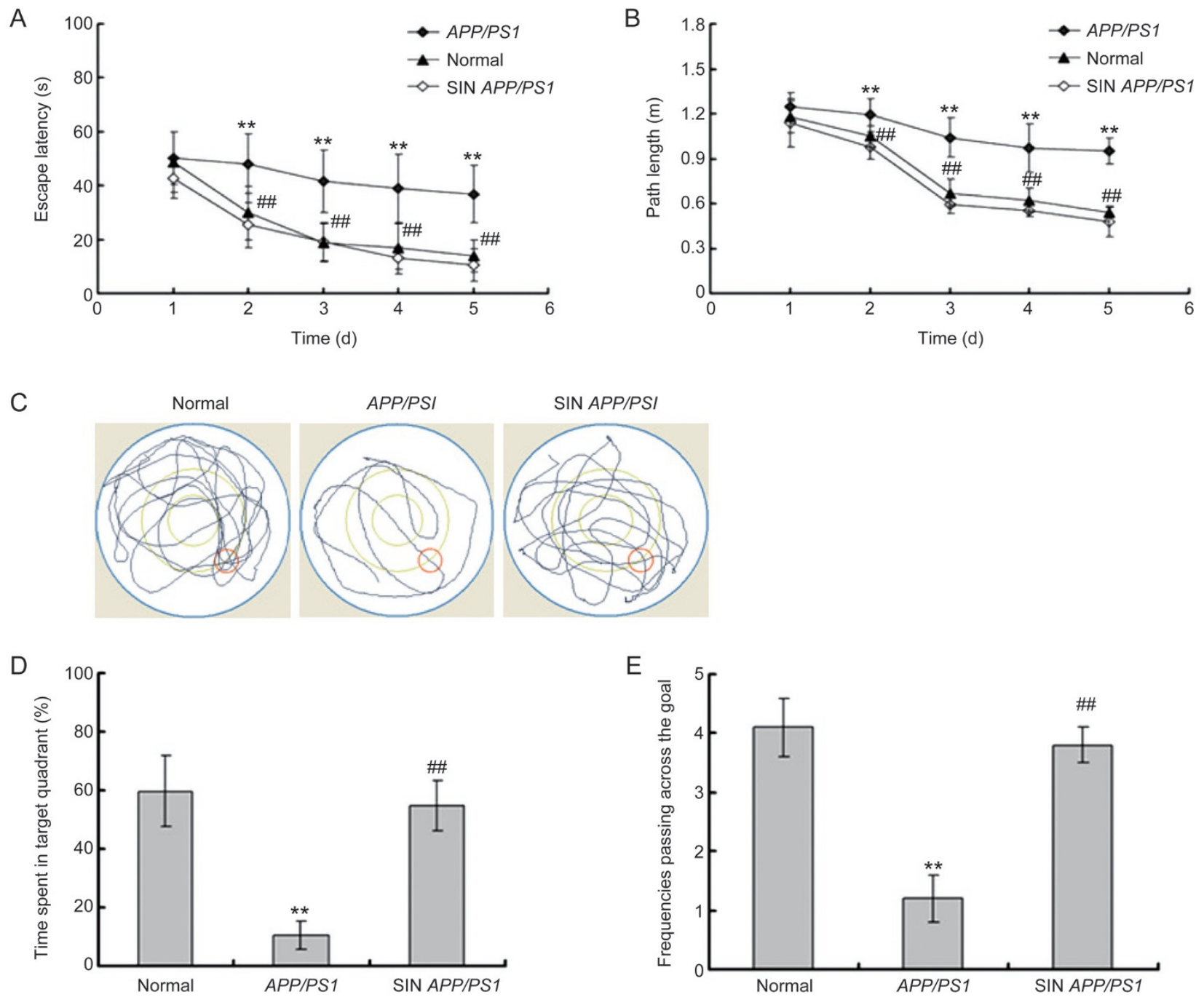

Figure 2. Schisandrin (SIN) treatment improved the escape latency (A) and path length (B) in the navigation test and improved performance of the probe trial in MWM of APP/PS1 mice $(n=10)$. (C) The representive locus plot after SIN treatment in the probe trial. (D) SIN treatment prolonged the time spent in target quadrant. (E) SIN treatment increased the frequencies of passing through the goal. ${ }^{* *} P<0.01$, compared with normal group; ${ }^{\# \#} P<0.01$, compared with APP/PS1 group.

ing and memory in APP/PS1 mice, the MWM behavioral test was performed following schisandrin treatment. In the navigation tests of the MWM, we observed on the first day that the normal mice, APP/PS1 mice and schisandrin-treated APP/PS1 mice had a similar escape latency $(P>0.05$, Figure $2 \mathrm{~A})$ and path length $(P>0.05$, Figure $2 B)$, which indicated that the motility or vision of mice was not affected before the navigation test and probe trial. In the navigation tests of the following days (from the second day to the fifth day), the APP/PS1 group showed a higher escape latency $(P<0.01$, Figure $2 \mathrm{~A})$, a longer path length $(P<0.01$, Figure $2 B)$ and slower improvement compared to the normal group. The escape latency and the path length in the schisandrin-treated APP/PS1 group in the navigation training were significantly improved compared to individuals in the APP/PS1 group $(P<0.01$, Figure $2 \mathrm{~A}$ and $\mathrm{B})$, which suggested that schisandrin treatment improved the impairment of the spatial acquisition of APP/PS1 mice. Furthermore, in the probe trial on the sixth day of MWM tests, the APP/PS1 mice passed through the original position of the platform fewer times $(P<0.01$, Figure $2 \mathrm{~A}$ and $\mathrm{C})$ and had a shorter stay in the target quadrant $(P<0.01$, Figure $2 \mathrm{D}$ and $\mathrm{E})$ compared to the normal group. In the schisandrin-treated APP/PS1 group, the time spent in the target quadrant $(P<0.01$, Figure $2 \mathrm{D}$ and $\mathrm{E})$ and the frequencies of passing the goal $(P<0.01$, Figure $2 \mathrm{~A}$ and C) significantly increased compared to the APP/PS1 group. The MWM test results indicated that schisandrin treatment improved the impairments of memory consolidation in APP/PS1 mice.

Thus, these results of behavioral tests demonstrated that schisandrin treatment ameliorated the cognitive impairment in spatial learning and memory function of APP/PS1 mice.

Schisandrin treatment attenuated $A \beta$ deposition in the $A P P / P S 1$ mouse brain

Immunohistochemical staining was used to determine the 
expressions of $A \beta$ in the hippocampus and cortex. No significant $A \beta$ accumulation was observed in the normal group (Figure $3 a)$. Figure $3 b$ shows that the $A \beta$ accumulation in the $A D$ model group was noticeable and that the optical density was significantly higher compared to the normal group. However, the schisandrin-treated APP/PS1 group exhibited obviously decreased $A \beta$ deposition (Figure $3 c$ ), which suggested that schisandrin treatment alleviated the overexpression of $A \beta_{(1-42)}$ in the hippocampus and cortex in AD model mice, which gave further evidence that schisandrin had anti-AD effects.

Schisandrin treatment adjusted NTs and their metabolites change in the brains of APP/PS1 transgenic mice according to UPLC-MS/MS determination

\section{Method validation}

The validation of linearity, accuracy (precision and trueness), sensitivity and selectivity was performed according to US Food and Drugs Administration (FDA) guidelines for bioanalytical assay validation.

\section{Linearity and LLOQ}

Calibration curves for the NTs and their metabolites in mouse brains were generated by plotting the peak area ratio $(y)$ of the analytes to the IS against nominal concentrations $(x)$ of the analytes in the standards by $1 / x^{2}$ weighted least square linear regressions. The standard calibration curves for the spiked brain tissue homogenates showed good linearity for the analytes. The results are shown in Table 2. The lower limits of quantification for each analyte in the brain tissue homogenates were $4.0 \mathrm{ng} / \mathrm{mL}$ for GABA, $1.0 \mathrm{ng} / \mathrm{mL}$ for DOPAC, HVA, DA, NE, 5-HT and 5-HIAA and $2.0 \mathrm{ng} / \mathrm{mL}$ for Glu and NE.

\section{Precision and accuracy}

The intra-day precision, inter-day precision and accuracy of the 9 analytes in the brain homogenates were validated, and the results were within the acceptable criteria (RSD\%: $<15 \%$; RE\%: $\pm 15 \%$ ). These results are represented in Table 3 , and they indicate that the method has acceptable precision and accuracy.

\section{Extraction recovery and matrix effects}

The mean extraction recoveries of the 9 analytes were greater than $85.0 \%$ at different concentration levels (Table 3 ), and the average extraction recovery of the IS was $93.2 \%$, which were acceptable values. The matrix effects of the analytes ranged from $90 \%$ to $110 \%$ at three concentration levels (Table 3), whereas the matrix effect of the IS was $91.9 \%$. These findings indicate that there was no significant difference in matrix effect for the analytes and IS.

\section{Stability}

The concentration measured for the 9 analytes at each of the
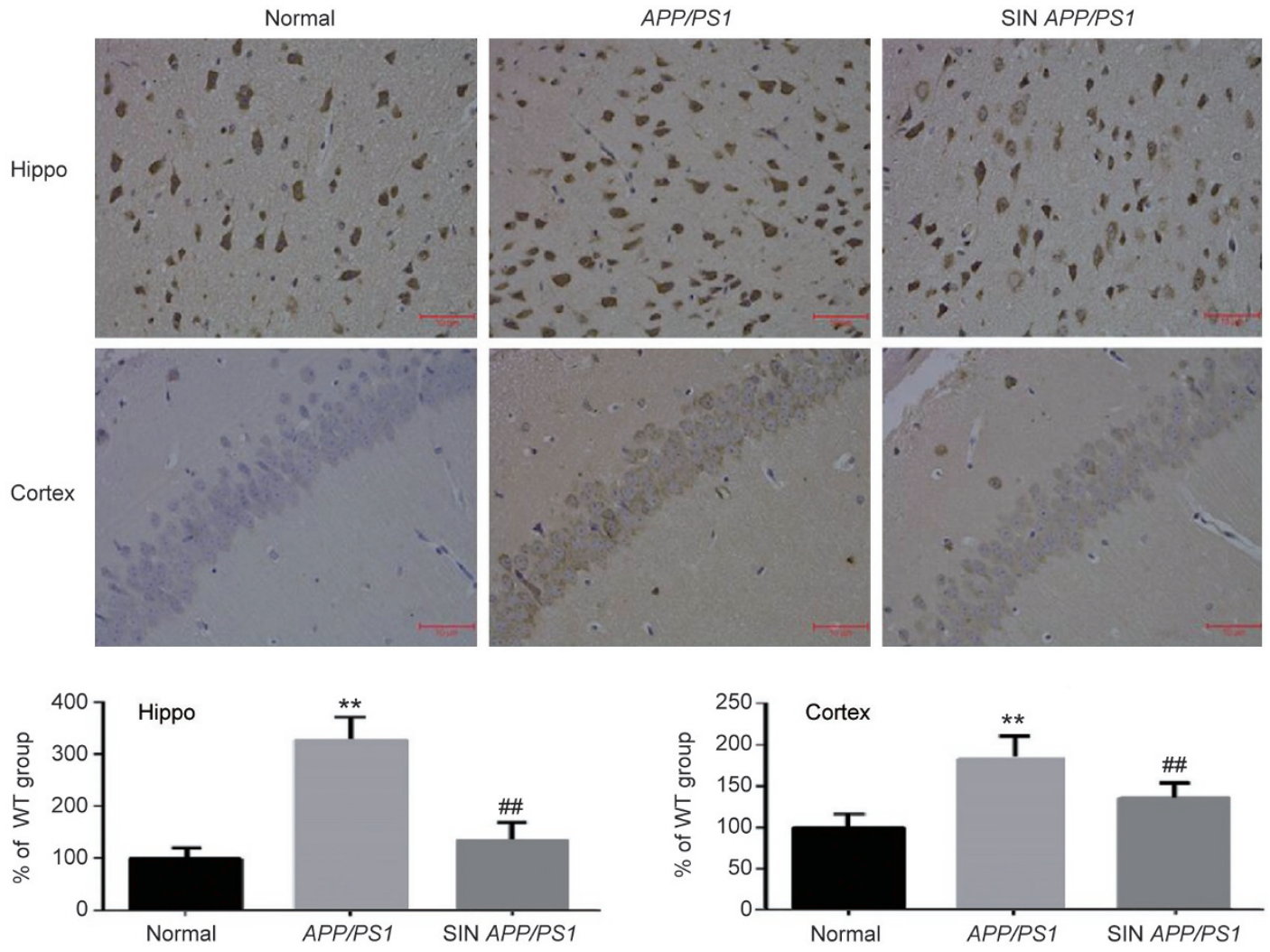

Figure 3. SIN treatment ameliorated the overexpression of $A \beta_{(1-42)}$ in the hippocampus and cortex of $A P P / P S 1$ mice by immunohistochemistry ( $\left.n=5\right)$. ${ }^{* *} P<0.01$ vs WT group. ${ }^{\# \#} P<0.01$ vs $A P P / P S 1$ group. 
Table 2. Linear ranges, regression equations and correlation coefficients of multi-components in mice brain biosamples.

\begin{tabular}{lclc}
\hline Analytes & Linear range $(\mathrm{ng} / \mathrm{mL})$ & Regression equation & Correlation coefficient $(r)$ \\
\hline GABA & $2.0-1000$ & $y=0.0023+0.0028 x$ & 0.9943 \\
Glu & $4.0-2000$ & $y=0.0265+0.023 x$ & 0.9927 \\
NE & $4.0-2000$ & $y=0.0013+0.0033 x$ & 0.9913 \\
DA & $1.0-500$ & $y=0.0026+0.0017 x$ & 0.9918 \\
5-HT & $1.0-500$ & $y=0.0019+0.0037 x$ & 0.9959 \\
5-HIAA & $1.0-500$ & $y=0.0015+0.0022 x$ & 0.9947 \\
ACh & $1.0-500$ & $y=0.0018+0.0026 x$ & 0.9987 \\
HVA & $1.0-500$ & $y=0.0025+0.0042 x$ & 0.9954 \\
DOPAC & $1.0-500$ & $y=0.0023+0.0076 x$ & 0.9963 \\
DHBA & $1.0-500$ & $y=0.0018+0.0031 x$ & 0.9952 \\
\hline
\end{tabular}

Table 3. Summary of accuracy, precision, recovery and matrix effect of the 9 analytes in mice brain $(n=6)$.

\begin{tabular}{|c|c|c|c|c|c|c|}
\hline Analytes & $\begin{array}{l}\text { Concentration } \\
(\mathrm{ng} / \mathrm{mL})\end{array}$ & $\begin{array}{l}\text { Intra-day } \\
\text { RSD (\%) }\end{array}$ & $\begin{array}{l}\text { Inter-day } \\
\text { RSD (\%) }\end{array}$ & $\begin{array}{c}\text { Accuracy } \\
\text { (RE \%) }\end{array}$ & $\begin{array}{c}\text { Recovery } \\
(\%, \text { mean } \pm \text { SD })\end{array}$ & $\begin{array}{l}\text { Matrix effect } \\
(\%, \text { mean } \pm S D)\end{array}$ \\
\hline \multirow[t]{3}{*}{ GABA } & 10 & 5.2 & 5.3 & 5.9 & $89.2 \pm 9.3$ & $88.9 \pm 5.1$ \\
\hline & 150 & 4.2 & 3.1 & 6.7 & $92.4 \pm 3.2$ & $90.2 \pm 1.4$ \\
\hline & 800 & 7.2 & 3.2 & -2.2 & $91.3 \pm 5.7$ & $92.8 \pm 1.0$ \\
\hline \multirow[t]{3}{*}{ Glu } & 20 & 8.3 & 4.9 & -8.7 & $85.5 \pm 3.2$ & $89.2 \pm 3.1$ \\
\hline & 300 & 5.4 & 4.7 & -3.1 & $94.2 \pm 1.3$ & $92.6 \pm 6.4$ \\
\hline & 1600 & 6.3 & 4.9 & 4.4 & $87.5 \pm 3.1$ & $88.2 \pm 4.1$ \\
\hline \multirow[t]{3}{*}{ NE } & 20 & 5.2 & 4.9 & -3.1 & $91.2 \pm 3.1$ & $92.1 \pm 4.5$ \\
\hline & 300 & 4.3 & 6.1 & 2.9 & $89.5 \pm 2.5$ & $92.8 \pm 5.5$ \\
\hline & 1600 & 5.1 & 4.3 & -7.2 & $95.2 \pm 3.2$ & $91.3 \pm 2.9$ \\
\hline \multirow[t]{3}{*}{ DA } & 5.0 & 5.3 & 6.1 & -6.2 & $90.2 \pm 1.0$ & $90.9 \pm 4.3$ \\
\hline & 750 & 5.9 & 6.9 & 7.1 & $86.4 \pm 2.9$ & $97.1 \pm 3.2$ \\
\hline & 400 & 2.1 & 3.6 & -2.2 & $92.3 \pm 4.8$ & $89.1 \pm 6.5$ \\
\hline \multirow[t]{3}{*}{ 5-HT } & 5.0 & 3.5 & 5.4 & -6.2 & $91.2 \pm 1.9$ & $92.3 \pm 4.2$ \\
\hline & 750 & 4.1 & 3.2 & -1.8 & $95.3 \pm 3.7$ & $94.8 \pm 3.0$ \\
\hline & 400 & 5.4 & 5.3 & -2.9 & $88.5 \pm 4.5$ & $92.8 \pm 3.5$ \\
\hline \multirow[t]{3}{*}{ 5-HIAA } & 5.0 & 5.2 & 7.2 & 5.9 & $87.2 \pm 3.1$ & $86.2 \pm 1.4$ \\
\hline & 750 & 5.8 & 5.1 & 1.2 & $88.7 \pm 8.3$ & $91.3 \pm 4.5$ \\
\hline & 400 & 6.7 & 5.2 & 2.2 & $89.2 \pm 1.9$ & $86.4 \pm 2.9$ \\
\hline \multirow[t]{3}{*}{$\mathrm{ACh}$} & 5.0 & 4.4 & 2.3 & -2.8 & $92.1 \pm 2.1$ & $92.3 \pm 4.8$ \\
\hline & 750 & 2.2 & 4.7 & 5.7 & $88.3 \pm 6.7$ & $91.2 \pm 1.9$ \\
\hline & 400 & 2.9 & 3.4 & -5.9 & $91.2 \pm 2.5$ & $88.2 \pm 5.5$ \\
\hline \multirow[t]{3}{*}{ DOPAC } & 5.0 & 4.7 & 2.3 & -2.9 & $87.7 \pm 1.3$ & $89.1 \pm 5.5$ \\
\hline & 750 & 2.5 & 4.6 & -4.1 & $97.1 \pm 3.2$ & $93.1 \pm 4.2$ \\
\hline & 400 & 2.7 & 4.4 & -5.1 & $89.1 \pm 6.5$ & $91.1 \pm 2.5$ \\
\hline \multirow[t]{3}{*}{ HVA } & 5.0 & 4.8 & 4.4 & -3.2 & $92.3 \pm 4.2$ & $89.9 \pm 8.2$ \\
\hline & 750 & 5.1 & 5.4 & -2.1 & $93.4 \pm 4.8$ & $92.2 \pm 5.0$ \\
\hline & 400 & 3.4 & 4.1 & -2.8 & $92.8 \pm 1.0$ & $90.6 \pm 2.8$ \\
\hline
\end{tabular}

QC levels deviated by $11.0 \%$, which demonstrated that they were stable in biosamples stored at room temperature for $24 \mathrm{~h}$, at $-20^{\circ} \mathrm{C}$ for at least 15 days, after three freeze and thaw cycles, and at $4{ }^{\circ} \mathrm{C}$ in the autosampler for $8 \mathrm{~h}$ after preparation. This outcome is well within acceptable limits and is described in Table 4.

\section{Method application}

The method described in this study was used to determine the level of NTs in mouse brains. The MS/MS spectra of these analytes are shown in Figure 1. Additionally, Figure 4 depicts the MRM chromatograms corresponding to the 9 analytes and the IS. The concentrations of the 9 NTs in the brain samples from the 3 groups are shown in Figure 5. The results show that the APP/PS1 model exhibited significant decreases in the brain levels of GABA, ACh, 5-HT, 5-HIAA, NE and DA and the metabolites DOPAC and HVA $(P<0.05)$ as well as a significant increase in the Glu levels $(P<0.05)$. However, schisandrin 
Table 4. Stability of the 9 analytes in mice brain $(n=3)$.

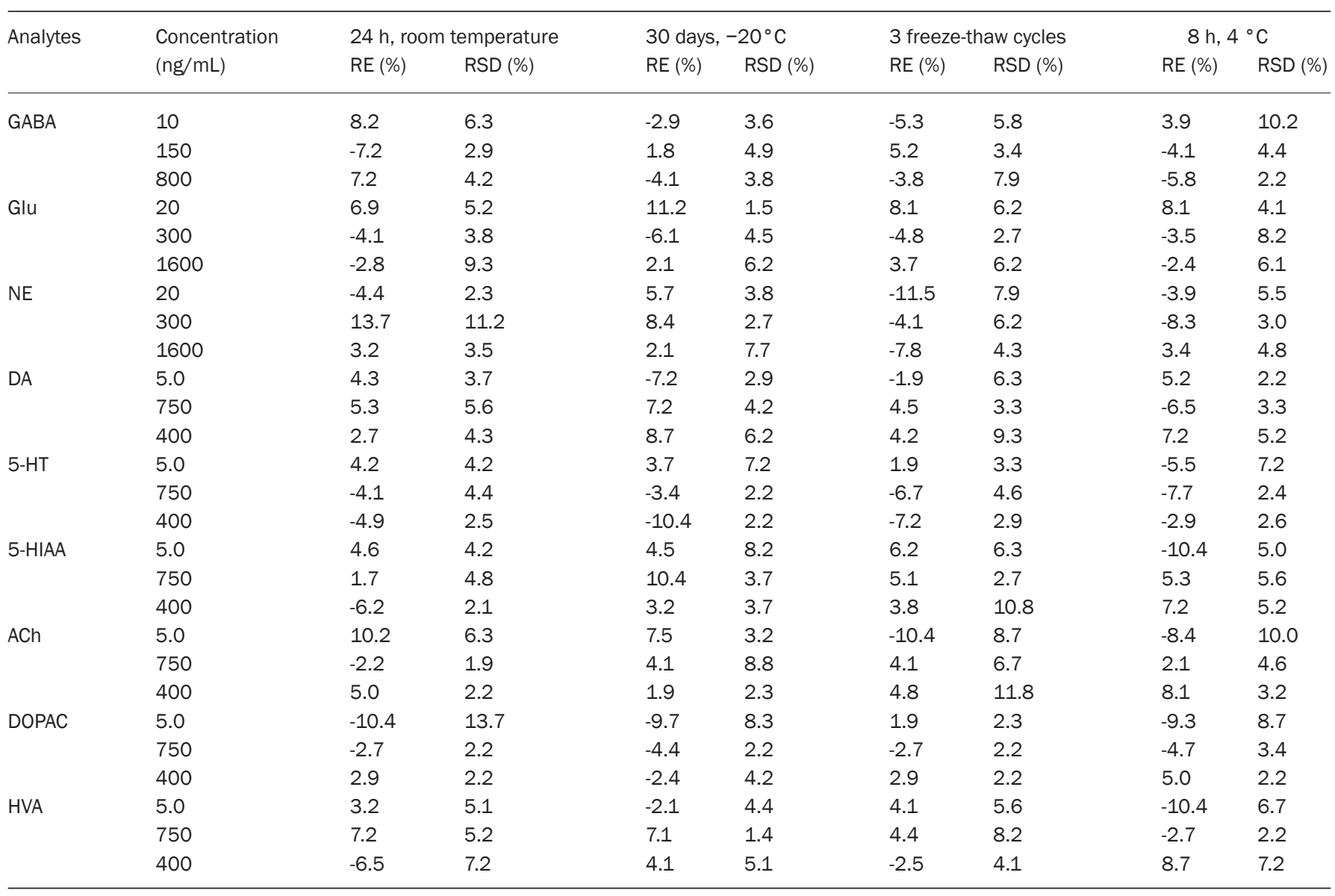

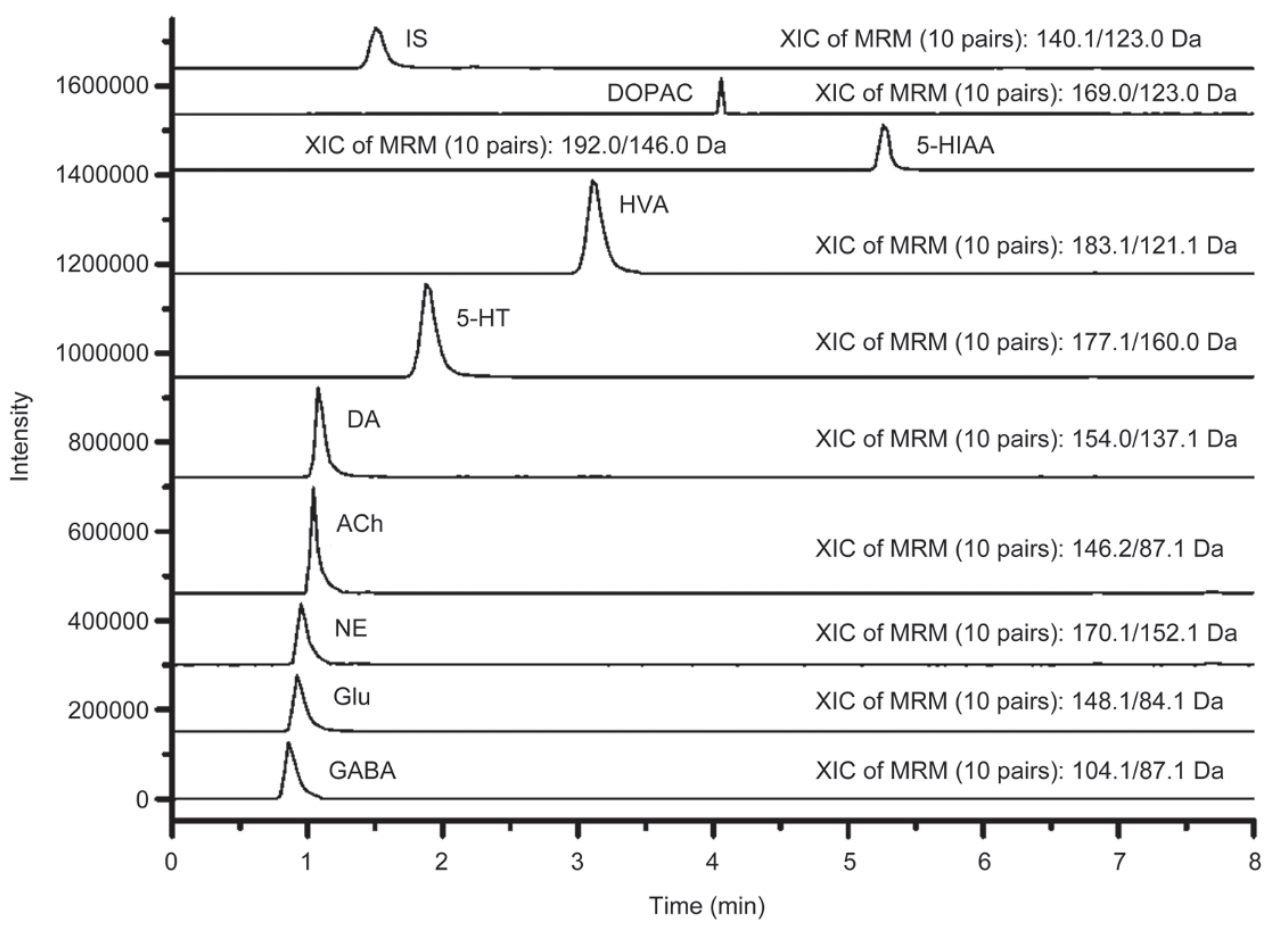

Figure 4. Typical chromatograms of the signals of NTs and their metabolites, together with DHBA in mice brain samples. Representative MRM chromatograms of GABA, Glu, NE, ACh, DA, 5-HT, HVA, 5-HIAA, DOPAC, and DHBA (IS). 


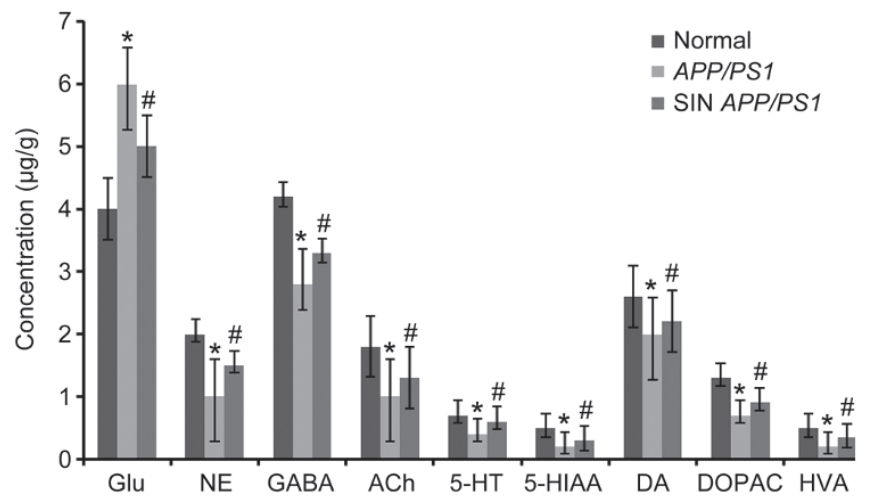

Figure 5. Levels of 9 NTs in mice brain normal group, AD model group, SIN-treated group. Results are expressed as mean \pm SEM $(n=10$ in each group). ${ }^{*} P<0.05$ vs the normal group. ${ }^{\#} P<0.05$ vs the $A D$ model group.

treatment significantly reversed these alterations. The results indicate that schisandrin could restore the dysfunction of various NTs in AD model mice.

\section{Discussion}

In this study, APP/PS1 transgenic mice were used as a mouse model of $\mathrm{AD}$, and model animals showed decreases in learning and memory abilities as well as deposition of $A \beta$ and other AD-like lesions. The results of the study showed that, compared to AD model mice, the effects on the learning and memory abilities of schisandrin-treated AD model mice were significantly ameliorated and the damage of $A \beta$ deposition in the hippocampus and cortex was attenuated, which indicated that the AD model was effective for drug evaluation. From our MWM test results, we found that schisandrin improved learning and memory in APP/PS1 mice, and the results indicated that schisandrin treatment ameliorated the impairments of memory consolidation in AD mice. From the immunohistochemistry test results, we found that schisandrin treatment alleviated the overexpression of $A \beta$ in the hippocampus and cortices in AD model mice, which further confirmed the therapeutic effects of schisandrin on $\mathrm{AD}$.

Previous reports indicated that NTs play a significant role in transmitting signals and that pathological alterations in NTs contribute to cognitive impairments and behavioral deficits in $\mathrm{AD}^{[29]}$. Glu, which is a type of excitatory neurotransmitter in the brain, is involved in acute or chronic neurodegenerative processes and neuropsychiatric disorders, including AD and Parkinson's disease ${ }^{[30]}$. Increased concentrations of Glu in AD might be a result of glial dysfunction, and schisandrin decreased the uptake of Glu, which led to an anti-AD effect. GABA is an important inhibitory neurotransmitter in the central nervous system. Increased concentrations of GABA are associated with greater cognitive improvement in $\mathrm{AD}^{[31]}$. $\mathrm{ACh}$ is an important neurotransmitter of the cholinergic system. Significant reductions in ACh levels have been correlated with behavioral symptoms of AD. Prior research has shown that 5-HT and its metabolite 5-HIAA are associated with aggressive behavior, and the concentrations of 5-HT and 5-HIAA in the brain tissue of $\mathrm{AD}$ patients were found to be lower compared to healthy individuals. DA and its metabolites DOPAC and HVA are synthesized in mesencephalon neurons, and significant differences were observed in the hippocampus among the $\mathrm{AD}$ and normal groups ${ }^{[32]}$. In our tests, we established a rapid and sensitive UPLC-MS/MS method for simultaneous determination of the 9 NTs in mouse brains, and this approach conformed to the methodology validation requirements. The test results showed that there was a significant difference in the NT levels in the brain between the APP/PS1 mice and normal mice $(P<0.05)$. After schisandrin treatment for the APP/ PS1 mice, there was significant restoration of the dysfunction of the NT levels. The determination results were consistent with results from the literature. These results suggested that schisandrin could alter the levels of these brain NTs and their metabolites to play a role in $\mathrm{AD}$ treatment.

Taking these results into consideration, schisandrin could restore the dysfunction of NTs, which was a possible mechanism for schisandrin in AD mice because the anti-AD effect of schisandrin was demonstrated through amelioration of learning and memory impairment and attenuation of $A \beta$ deposition. Therefore, this study provides a new strategy for $A D$ treatment using schisandrin.

In summary, we found that schisandrin could ameliorate learning and memory impairment and attenuate $A \beta$ deposition in AD model mice. A possible mechanism was also explored. Therefore, an efficient, sensitive and selective UPLC-MS/MS analysis was developed and validated, and it was successfully applied to the simultaneous determination of NTs in the brains of AD model mice. The possible mechanism of the anti-AD effect involved restoring the dysfunction of these NTs. All these findings provide scientific evidence that schisandrin could be developed as an effective treatment for AD.

\section{Acknowledgements}

The work was supported by the National Natural Science Foundation of China (№ 81703519) and the Doctor Startup Science Foundation of Liaoning Province (№ 20170520257).

\section{Author contribution}

Bin-bin WEI conceived and designed the experiments; Bin-bin WEI and Zai-xing CHEN performed the experiments; Mingyan LIU analyzed the data; Bin-bin WEI wrote the paper; Minjie WEI reviewed the paper. All authors read and approved the final manuscript.

\section{Abbreviations}

$\mathrm{AD}$, Alzheimer's disease; $\mathrm{A} \beta, \beta$-amyloid peptide; NTs, neurotransmitters; CNS, central nervous system; TCM, Traditional Chinese Medicine; MWM, Morris water maze; ESI, electrospray ionization; UPLC, ultra-high-performance liquid chromatography; LLOQ, lower limit of quantification; SD, standard deviation.

\section{References}

1 Querfurth HW, Laferla FM. Alzheimer's Disease. N Eng J Med 2010; 
362: 329-44.

2 O'Brien RJ, Wong PC. Amyloid precursor protein processing and Alzheimer's disease. Annu Rev Neurosci 2011; 34: 185-204.

3 Rafii MS, Aisen PS. Advances in Alzheimer's disease drug development. BMC Med 2015; 13: 62-70.

4 Supnet C, Bezprozvanny I. Presenilins function in ER calcium leak and Alzheimer's disease pathogenesis. Cell Calcium 2011; 50: 303-9.

5 Graham SF, Chevallier OP, Roberts D, Hölscher C, Elliott CT, Green BD. Investigation of the human brain metabolome to identify potential markers for early diagnosis and therapeutic targets of Alzheimer's disease. Anal Chem 2013; 85: 1803-11.

6 Tanzi RE, Bertram L. Twenty years of the Alzheimer's disease amyloid hypothesis: a genetic perspective. Cell 2005; 120: 545-55.

7 Nizzari M, Thellung S, Corsaro A, Florio T. Neurodegeneration in Alzheimer disease: role of amyloid precursor protein and presenilin 1 intracellular signaling. J Toxicol 2012; 2012: 187297-302.

8 Prakash A, Kalra J, Mani V, Ramasamy K, Majeed AA. Pharmacological approaches for Alzheimer's disease: neurotransmitter as drug targets. Expert Rev Neurother 2015; 15: 53-71.

9 Chen KH, Reese EA, Kim HW, Rapoport SI, Rao JS. Disturbed neurotransmitter transporter expression in Alzheimer's disease brain. J Alzheimers Dis 2011; 26: 755-66.

10 Limon A, Reyes-Ruiz JM, Miledi R. Loss of functional GABA(A) receptors in the Alzheimer diseased brain. Proc Natl Acad Sci U S A 2012; 109: 10071-6.

11 Kovac A, Somikova Z, Zilka N, Novak M. Liquid chromatographytandem mass spectrometry method for determination of panel of neurotransmitters in cerebrospinal fluid from the rat model for tauopathy. Talanta 2014; 119: 284-90.

12 Gong YG, Liu Y, Zhou L, Di X, Li W, Li Q, et al. A UHPLC-TOF/MS method based metabonomic study of total ginsenosides effects on Alzheimer disease mouse model. J Pharm Biomed Anal 2015; 115: 174-82.

13 Greco S, Danysz W, Zivkovic A, Gross R, Stark H. Microdialysate analysis of monoamine neurotransmitters-a versatile and sensitive LC-MS/MS method. Anal Chim Acta 2013; 771: 65-72.

14 Prokšelj T, Jerin A, Muck-Seler D, Kogoj A. Decreased platelet serotonin concentration in Alzheimer's disease with involuntary emotional expression disorder. Neurosci Lett 2014; 578: 71-4.

15 Parsons CG, Danysz W, Dekundy A, Pulte I. Memantine and cholinesterase inhibitors: complementary mechanisms in the treatment of Alzheimer's disease. Neurotox Res 2013; 24: 358-69.

16 Burns A, Bernabei R, Bullock R. Safety and efficacy of galantamine (Reminyl) in severe Alzheimer's disease (the SERAD study): a randomised, placebo-controlled, double-blind trial. Lancet Neurol
2009; 8: 39-45.

17 Birks J, Grimley EJ, lakovidou V. Rivastigmine for Alzheimer's disease. Cochrane DB Syst Rev 2009; 15: 191-201.

18 Minarini A, Milelli A, Simoni E. Multifunctional tacrine derivatives in Alzheimer's disease. Curr Top Med Chem 2013; 13: 765-71.

19 Yang YH, Chen $\mathrm{CH}$, Chou MC. Concentration of donepezil to the cognitive response in Alzheimer disease. J Clin Psychopharmacol 2013; 33: 351-61.

$20 \mathrm{Yu} \mathrm{Z}$, Wei C. Research status on treating AD in the integrative medicine. Clin J Chin Med 2012; 4: 115-9.

21 Wang XM, Liu MX, Zhang Y. Pharmacological research of Schisandra chinensis (Turcz.) Baill. North Pharm 2013; 10: 72-8.

22 Shi L, Wang ZC, Femg XQ. Advances in studies on chemical constituents and pharmacological activities of Schisandrae Chinensis. Drug Eval Res 2011; 34: 208-12.

23 Jeong EJ, Lee HK, Lee KY, Jeon BJ, Kim DH, Park JH, et al. The effects of lignan-riched extract of Shisandra chinensis on amyloid- $\beta$-induced cognitive impairment and neurotoxicity in the cortex and hippocampus of mouse. J Ethnopharmacol 2013; 146: 347-54.

24 Jankowsky JL, Slunt HH, Ratovitski T, Jenkins NA, Copeland NG, Borchelt DR. Co-expression of multiple transgenes in mouse CNS: a comparison of strategies. Biomol Eng 2001; 17: 157-65.

25 Van TG, Kiliaan AJ, Kadish I. Deposition of mouse amyloid beta in human APP/PS1 double and single AD model transgenic mice. Neurobiol Dis 2006; 23: 653-62.

26 Vorhees CV, Williams MT. Morris water maze: procedures for assessing spatial and related forms of learning and memory. Nat Protoc 2006; 1: 848-58.

27 http://www.fda.gov/cder/guidance/index.htm (Guidance for Industry, bioanalytical Method Validation. updated 2001).

28 ICH Harmonised Tripartite Guideline, Validation of Analytical Procedure: Methodology, International Conference on Harmonisation of Technical Requirements for Registration of Pharmaceuticals for Human Use, Geneva, 1996.

29 Strac DS, Muck-Seler D, Pivac N. Neurotransmitter measures in the cerebrospinal fluid of patients with Alzheimer's disease: a review. Psychiatr Danub 2015; 27: 14-24.

30 Kallarackal AJ, Kvarta MD, Cammarata E, Jaberi L, Cai X, Bailey AM, et al. Chronic stress induces a selective decrease in AMPA receptormediated synaptic excitation at hippocampal temporoammonic-CA1 synapses. J Soc Neurosci 2013; 33: 15669-74.

31 Solas M, Puerta E, Ramirez MJ. Treatment options in Alzheimer's disease: the GABA story. Curr Pharm Des 2015; 21: 4960-71.

32 Martorana A, Koch G. Is dopamine involved in Alzheimer's disease? Front Aging Neurosci 2014; 6: 453-65. 